\title{
Die wirtschaftliche Bedeutung der Freizügigkeit aus polnischer Sicht
}

\author{
Maciej Żukowski
}

A. Arbeitsmigration aus wirtschaftswissenschaftlicher Perspektive

B. Arbeitsmigration innerhalb der EU

C. Arbeitsmigration aus Polen seit 2004

D. Gewinne und Kosten aus polnischer Sicht

E. Bilanz und Perspektiven

\section{A. Arbeitsmigration aus wirtschaftswissenschaftlicher Perspektive}

Im Lichte der ökonomischen Theorie wird Migration als Phänomen der Mobilität des Produktionsfaktors Arbeit begriffen. Migration folgt wesentlich dem Lohnabstand zwischen der Zuwanderungs- und der Abwanderungsregion. Der Einzelne stellt dabei den Nutzen einer Auswanderung deren Kosten gegenüber. Alle Modelle stützen sich auf die Vorstellung, dass der Migrant eine Migrationsentscheidung rational trifft.

Neben dem höheren Einkommen als wichtigstem Faktor besteht der Nutzen u.a. in den eng damit verbundenen besseren Lebensbedingungen, höheren Lebenschancen und besseren Umweltbedingungen. Die Kosten bestehen u.a. in der geographischen Entfernung, den sprachlichen und kulturellen Unterschieden sowie den fehlenden sozialen Netzwerken. Ein Anreiz zur Auswanderung entsteht, wenn eine individuelle rationelle Gegenüberstellung von Nutzen und Kosten ein entsprechendes positives Nettoergebnis erwarten lässt. So entsteht allerdings erst ein Migrationspotenzial. Denn nur ein Teil derjenigen, die einen Anreiz zur Auswanderung hätten, entscheidet sich wirklich für diesen Schritt.

Natürlich kann Migration auch primär politisch bedingt sein, wenn z.B. Kriege oder Diktaturen Menschen dazu bewegen, ihr Land zu verlassen und Exil zu suchen. Dieser Text beschränkt sich jedoch auf die Arbeitsmigration, die auch im Kontext der Freizügigkeit innerhalb der EU eine entscheidende Rolle spielt. 
Die Sogtheorie erklärt am Push-and-Pull-Modell das Zustandekommen des Migrationsdrucks aus dem Gefälle zwischen zwei Ländern. Zu den Pushfaktoren im Herkunftsland (Auswanderungsland, Ursprungsland) zählen z.B. hohe Arbeitslosigkeit, ein niedriges Lohnniveau, eine instabile politische Situation oder schlechte Umweltbedingungen. Die Pullfaktoren dagegen gehen von den potentiellen Aufnahmeländern (Einwanderungsländern, Zielstaaten) aus und bestehen v.a. in einem höheren Verdienst, besseren Arbeitsbedingungen oder besseren Karrierechancen. Die Unterscheidung ist nicht eindeutig - ein erhoffter höherer Verdienst im Zielstaat ist ein Pullfaktor, was aber bedeutet, dass ein niedriger Verdienst im Heimatland als Pushfaktor wirkt.

Die Demographie spielt ebenfalls eine wichtige Rolle für die Auswanderungsentscheidung. Da tendenziell jüngere Menschen dazu bereit sind, auszuwandern, ist ein relativ hoher Anteil von Menschen dieser Altersgruppe an der Gesamtbevölkerung eine wesentliche Determinante des Migrationspotentials.

Neben der traditionellen endgültigen Auswanderung, die nach wie vor eine vorherrschende Migrationsausprägung ist, entwickeln sich auch neue Formen der transnationalen Mobilität wie die Pendelwanderung, entweder in zyklischen Formen (z.B. als Tagespendler) oder mit einer unregelmäßigen Periodizität. Diese Formen sind auch im Kontext der EU-Erweiterung von besonderer Bedeutung.

Aus globaler Perspektive führt Migration zu einem produktiveren Einsatz des Faktors Arbeit und damit zu einem Anstieg des globalen Sozialprodukts. Somit ist Migration langfristig sowohl für die Aus- als auch für die Zuwanderungsländer günstig.

Allerdings sind die Gewinne und Kosten der Migration zunächst nicht gleich über die Ein- und Auswanderungsländer und die verschiedenen Personengruppen verteilt.

\section{B. Arbeitsmigration innerhalb der EU}

Vom Beginn der europäischen Integration an wurde die Freizügigkeit der Arbeitnehmer als eine von vier Grundfreiheiten anerkannt und im EU-Recht fest verankert. So wurden Regelungen eingeführt, die der Verwirklichung der Freizügigkeit dienen sollen, u.a. das Koordinierungsrecht der sozialen Sicherung. Um die Inanspruchnahme der Freizügigkeit zu fördern, sind auch mehrere Institutionen geschaffen worden, wie das Europäische Job-Netzwerk EURES. Man kann also sagen, dass alles getan wurde, um Barrieren abzubauen und die Rahmenbedingungen für die Arbeitsmigration optimal zu gestalten.

Für die EU-Bürger ist die Freizügigkeit sehr wichtig. Die Reisefreiheit wird von den meisten als die wichtigste Komponente der EU angesehen. Im neuesten Eurobarometer 76 vom November 2011 wurde die Frage „Was bedeutet die EU für Sie persönlich?“ von $41 \%$ aller Befragten in EU-27 (54\% in Deutschland und 45\% in Polen) so beant- 
wortet: „Die Freiheit, überall innerhalb der EU reisen, studieren und arbeiten zu können"1.

Allerdings bedeutet der hohe Wert, den die EU-Bürger der Freizügigkeit beimessen, nicht, dass auch viele Bürger von der Möglichkeit tatsächlich Gebrauch machen: Während Reisen zur Selbstverständlichkeit geworden sind, ist Wohnen und Arbeiten in einem anderen EU-Mitgliedstaat eher eine Seltenheit geblieben. Die Mobilität von Arbeitnehmern ist innerhalb der EU sehr gering, insbesondere im Vergleich mit der Mobilität von Waren, Dienstleistungen und Kapital. Dies lässt sich mit den Annahmen der ökonomischen Theorie leicht erklären. Kurz gefasst: Der Nutzen der Migration ist kleiner geworden, während die Kosten hoch geblieben sind.

Im Zuge der erfolgreichen europäischen Integration wurden die früheren Einkommensunterschiede weitgehend abgebaut. Das ist die wichtigste Erklärung der aktuell niedrigen Mobilität innerhalb der EU (Tabelle 1). Man kann also feststellen, dass die europäische Integration zwar einerseits migrationsfördernd wirkt, indem Migrationsbarrieren abgebaut werden, andererseits jedoch langfristig tatsächlich Anti-Migrationswirkungen erzeugt, indem sie zur Konvergenz, d.h. einer größeren Annäherung führt.

Auf der anderen Seite bestehen bestimmte Barrieren wie mangelnde Sprachkenntnisse, der Verlust von sozialen Netzwerken oder soziale und kulturelle Unterschiede fort.

Tabelle 1: Bruttoinlandsprodukt (BIP) in Kaufkraftstandards (KKS), EU-27=100, 1995 und 2010

\begin{tabular}{|l|c|c|}
\hline Staat & $\mathbf{1 9 9 5}$ & $\mathbf{2 0 1 0}$ \\
\hline Belgien & 128 & 119 \\
\hline Bulgarien & 32 & 44 \\
\hline Dänemark & 131 & 127 \\
\hline Deutschland & 129 & 118 \\
\hline Estland & 36 & 64 \\
\hline Finnland & 107 & 115 \\
\hline Frankreich & 116 & 108 \\
\hline Griechenland & 84 & 90 \\
\hline Irland & 103 & 128 \\
\hline Italien & 121 & 101 \\
\hline Lettland & 31 & 51 \\
\hline Litauen & 35 & 57 \\
\hline Luxemburg & 223 & 271 \\
\hline Malta & 86 & 83 \\
\hline Niederlande & 123 & 133 \\
\hline
\end{tabular}

1 European Commission, Standard Eurobarometer 76, Table of results, Die Öffentliche Meinung in der Europäischen Union. Befragung: November 2011, Veröffentlichung: Dezember 2011, S. 50. 


\begin{tabular}{|l|c|c|}
\hline Österreich & 134 & 126 \\
\hline Polen & 43 & 63 \\
\hline Portugal & 77 & 80 \\
\hline Rumänien & 33 & 46 \\
\hline Schweden & 125 & 123 \\
\hline Slowenien & 74 & 85 \\
\hline Slowakei & 47 & 74 \\
\hline Spanien & 91 & 100 \\
\hline Tschechische Republik & 77 & 80 \\
\hline Ungarn & 51 & 65 \\
\hline Vereinigtes Königreich & 113 & 112 \\
\hline Zypern & 87 & 99 \\
\hline
\end{tabular}

Quelle: Eurostat Datenbank, Zugang am 10.03.2012, http://epp.eurostat.ec.europa.eu/tgm/ printTable.do?tab $=$ table $\&$ plugin $=1 \&$ language $=$ de $\&$ p $\operatorname{code}=$ tec $00114 \&$ printPreview $=$ true .

Ein Einkommensunterschied von 60-70\% wie zwischen dem (abgesehen vom kleinen Luxemburg) reichsten alten EU-Mitgliedstaat Niederlande und dem ärmsten alten EU-Mitgliedstaat Portugal scheint nicht mehr ausreichend zu sein, um die Kosten (Sprache, Klima, Kultur, ...) zu kompensieren und deutliche Migrationsbewegungen auszulösen. Die Unterschiede zwischen den alten und den neuen EU-Mitgliedstaaten sind noch größer und haben so erwartungsgemäß eine Migrationswelle ausgelöst. Auf der anderen Seite dokumentiert aber auch Tabelle 1 eine klare Konvergenz, die zur Senkung des Migrationsdrucks zwischen den neuen und den alten EU-Mitgliedstaaten führen wird. Auf längere Sicht ist also auch die EU-Erweiterung „die beste AntiMigrationspolitik“2.

2010 bestanden ungefähr 6,5\% der Bevölkerung der Mitgliedstaaten aus Ausländern. Dabei war der Anteil der Bürger von außerhalb der EU gelegenen Staaten $(4,0 \%)$ deutlich größer als die der EU-Bürger (2,5\%) (Tabelle 2). Die Statistiken zeigen also, dass nach über 50 Jahren der europäischen Integration lediglich 2,5\% der EU-Bürger die Arbeitnehmerfreizügigkeit voll in Anspruch nehmen.

2 Straubhaar, East-West Migration: Will it be a Problem?, Intereconomics July/August 2001, S. 167170, 167. 
Tabelle 2: Ausländer in den Mitgliedstaaten der EU im Jahr 2010

\begin{tabular}{|c|c|c|c|c|c|c|c|}
\hline \multirow[t]{3}{*}{ Staat } & \multirow{3}{*}{$\begin{array}{l}\text { Bevölkerung } \\
\quad(1000)\end{array}$} & \multicolumn{6}{|c|}{ Ausländer } \\
\hline & & \multicolumn{2}{|c|}{ Insgesamt } & \multicolumn{2}{|c|}{ EU-Bürger } & \multicolumn{2}{|c|}{ Nicht-EU-Bürger } \\
\hline & & 1000 & $\%$ & 1000 & $\%$ & 1000 & $\%$ \\
\hline EU-27 & 501098,0 & 32493,2 & 6,5 & 12336,0 & 2,5 & 20157,2 & 4,0 \\
\hline Belgien & 10839,9 & 1052,8 & 9,7 & 715,1 & 6,6 & 337,7 & 3,1 \\
\hline Bulgarien & 7563,7 & & & & & & \\
\hline Dänemark & 5534,7 & 329,8 & 6,0 & 115,5 & 2,1 & 214,3 & 3,9 \\
\hline Deutschland & 81802,3 & 7130,9 & 8,7 & 2546,3 & 3,1 & 4584,7 & 5,6 \\
\hline Estland & 1340,1 & 212,7 & 15,9 & 11,0 & 0,8 & 201,7 & 15,1 \\
\hline Finnland & 5351,4 & 154,6 & 2,9 & 56,1 & 1,0 & 98,5 & 1,8 \\
\hline Frankreich & 64716,3 & 3769,0 & 5,8 & 1317,6 & 2,0 & 2451,4 & 3,8 \\
\hline Griechenland & 11305,1 & 954,8 & 8,4 & 163,1 & 1,4 & 791,7 & 7,0 \\
\hline Irland & 4467,9 & 384,4 & 8,6 & 309,4 & 6,9 & 75,0 & 1,7 \\
\hline Italien & 60340,3 & 4235,1 & 7,0 & 1241,3 & 2,1 & 2993,7 & 5,0 \\
\hline Lettland & 2248,4 & 392,2 & 17,4 & 9,7 & 0,4 & 382,4 & 17,0 \\
\hline Litauen & 3329,0 & 37,0 & 1,1 & 2,4 & 0,1 & 34,6 & 1,0 \\
\hline Luxemburg & 502,1 & 215,7 & 43,0 & 186,2 & 37,1 & 29,5 & 5,9 \\
\hline Malta & 413,0 & 16,7 & 4,0 & 5,4 & 1,3 & 11,3 & 2,7 \\
\hline Niederlande & 16575,0 & 652,2 & 3,9 & 310,9 & 1,9 & 341,3 & 2,1 \\
\hline Österreich & 8367,7 & 876,4 & 10,5 & 328,3 & 3,9 & 548,0 & 6,5 \\
\hline Polen & 38167,3 & 45,5 & 0,1 & 14,8 & 0,0 & 30,7 & 0,1 \\
\hline Portugal & 10637,7 & 457,3 & 4,3 & 94,2 & 0,9 & 363,1 & 3,4 \\
\hline Rumänien & 21462,2 & . & . & . & . & . & . \\
\hline Schweden & 9340,7 & 590,5 & 6,3 & 265,8 & 2,8 & 324,7 & 3,5 \\
\hline Slowenien & 2047,0 & 82,2 & 4,0 & 4,6 & 0,2 & 77,6 & 3,8 \\
\hline Slowakei & 5424,9 & 62,9 & 1,2 & 38,7 & 0,7 & 24,2 & 0,4 \\
\hline Spanien & 45989,0 & 5663,5 & 12,3 & 2327,8 & 5,1 & 3335,7 & 7,3 \\
\hline $\begin{array}{l}\text { Tschechische } \\
\text { Republik }\end{array}$ & 10506,8 & 424,4 & 4,0 & 137,0 & 1,3 & 287,4 & 2,7 \\
\hline Ungarn & 10014,3 & 200,0 & 2,0 & 118,9 & 1,2 & 81,1 & 0,8 \\
\hline $\begin{array}{l}\text { Vereinigtes } \\
\text { Königreich }\end{array}$ & 62008,0 & 4367,6 & 7,0 & 1922,5 & 3,1 & 2445,1 & 3,9 \\
\hline Zypern & 803,1 & 127,3 & 15,9 & 83,5 & 10,4 & 43,8 & 5,5 \\
\hline
\end{tabular}

Quelle: Vasileva, Population and social conditions, Eurostat, Statistics in focus 34 (2011), S. 2. 
Die Zahlen zeigen allerdings nicht die gesamte Mobilität in Europa - sie berücksichtigen weder kurzfristige Arbeitsaufenthalte im Ausland für einige Monate noch ,crossborder commuters" oder Saisonarbeiter.

\section{Arbeitsmigration aus Polen seit 2004}

Polen ist traditionell ein (Netto-)Auswanderungsland. Insbesondere im 19. Jahrhundert begann die große Auswanderung aus den von den drei Teilungsmächten - Österreich, Preußen und Russland - besetzten polnischen Gebieten nach Amerika und in viele europäische Länder. Auch die Auswanderung nach Deutschland hat eine lange Tradition $^{3}$.

Nach dem zweiten Weltkrieg, als Polen Teil der sowjetischen Machtzone wurde, spielten neben den ökonomischen auch politische Argumente eine wichtige Rolle für die Auswanderung polnischer Staatsbürger. Dabei war Deutschland stets das wichtigste Zielland der polnischen Auswanderung innerhalb Europas.

Es war besonders die Angst vor der Zuwanderung aus Polen, einem großen und relativ armen Land, die zu Übergangsregelungen von sieben Jahren in den Beitrittsverträgen mit allen acht postkommunistischen Staaten, die sich der EU am 1. Mai 2004 anschlossen, führte. Die Angst war insbesondere in Deutschland groß, was aufgrund der Nähe, der Größe und der Migrationstraditionen vollkommen verständlich war.

Nur drei alte Mitgliedstaaten - das Vereinigte Königreich, Irland und Schweden haben sich dazu entschlossen, ihre Arbeitsmärkte bereits zum 1. Mai 2004 für die "Neuen", darunter Polen, zu öffnen. Diese Entscheidung hat die Zielrichtung der Auswanderung aus Polen wesentlich beeinflusst (Tabelle 3).

Tabelle 3: Auswanderung aus Polen, vorübergehender Aufenthalt, in den Jahren 2004 - $2010^{4}$

\begin{tabular}{|c|c|c|c|c|c|c|c|c|}
\hline & \multicolumn{8}{|c|}{ Zahl der Auswanderer in Tausend } \\
\hline & $2002^{5}$ & 2004 & 2005 & 2006 & 2007 & 2008 & 2009 & 2010 \\
\hline Insgesamt & 786 & 1000 & 1450 & 1950 & 2270 & 2210 & 1870 & 1990 \\
\hline Europa, davon & 461 & 770 & 1200 & 1610 & 1925 & 1887 & 1635 & 1690 \\
\hline EU (27 Länder) ${ }^{6}$, davon & 451 & 750 & 1170 & 1550 & 1860 & 1820 & 1570 & 1615 \\
\hline
\end{tabular}

3 Marek, Praca Polaków w Niemczech. Półtora wieku emigracji zarobkowej (Arbeit der Polen in Deutschland. Eineinhalb Jahrhunderte der Arbeitsauswanderung), Instytut Pracy i Spraw Socjalnych, 2008.

4 Die Zahlen beziehen sich auf Personen, die vorübergehend im Ausland wohnen, für 2002-2006 länger als 2 Monate, für 2007-2010 - länger als 3 Monate.

5 Unter Berücksichtigung der Wanderer ohne ein festgestelltes Aufenthaltsland betrug die Zahl der Wanderer im Jahr 2002 in den europäischen Ländern 547.000, in den EU-Ländern 535.000.

6 Bis 2006: 25 Länder. 
Die wirtschaftliche Bedeutung der Freizügigkeit aus polnischer Sicht

\begin{tabular}{|l|c|c|c|c|c|c|c|c|}
\hline Belgien & 14 & 13 & 21 & 28 & 31 & 33 & 34 & 45 \\
\hline Dänemark & $\cdot$ & $\cdot$ & $\cdot$ & $\cdot$ & 17 & 19 & 20 & 19 \\
\hline Deutschland & $\mathbf{2 9 4}$ & $\mathbf{3 8 5}$ & $\mathbf{4 3 0}$ & $\mathbf{4 5 0}$ & $\mathbf{4 9 0}$ & $\mathbf{4 9 0}$ & $\mathbf{4 1 5}$ & $\mathbf{4 5 5}$ \\
\hline Finnland & 0,3 & 0,4 & 0,7 & 3 & 4 & 4 & 3 & 3 \\
\hline Frankreich & 21 & 30 & 44 & 49 & 55 & 56 & 47 & 55 \\
\hline Griechenland & 10 & 13 & 17 & 20 & 20 & 20 & 16 & 16 \\
\hline Irland & 2 & 15 & 76 & 120 & 200 & 180 & 140 & 125 \\
\hline Italien & 39 & 59 & 70 & 85 & 87 & 88 & 85 & 92 \\
\hline Niederlande & 10 & 23 & 43 & 55 & 98 & 108 & 84 & 108 \\
\hline Österreich & 11 & 15 & 25 & 34 & 39 & 40 & 38 & 32 \\
\hline Portugal & 0,3 & 0,5 & 0,6 & 1 & 1 & 1 & 1 & 1 \\
\hline Schweden & 6 & 11 & 17 & 25 & 27 & 29 & 31 & 37 \\
\hline Spanien & 14 & 26 & 37 & 44 & 80 & 83 & 84 & 50 \\
\hline Tschechische Republik & $\cdot$ & $\cdot$ & $\cdot$ & $\cdot$ & 8 & 10 & 9 & 7 \\
\hline Vereinigtes Königreich & 24 & 150 & 340 & 580 & 690 & 650 & 555 & 560 \\
\hline Zypern & $\cdot$ & $\cdot$ & $\cdot$ & $\cdot$ & 4 & 4 & 3 & 3 \\
\hline $\begin{array}{l}\text { Länder außerhalb der } \\
\text { EU, davon }\end{array}$ & 10 & 20 & 30 & 60 & 65 & 67 & 65 & 75 \\
\hline Norwegen & $\cdot$ & $\cdot$ & $\cdot$ & $\cdot$ & 36 & 38 & 45 & 46 \\
\hline
\end{tabular}

Quelle: Główny Utząd Statystyczny Departament Badań Demograficznych, Informacja o rozmiarach i kierunkach emigracji z Polski w latach 2004 - 2010, 2011, Zugang am 29.03.2012,

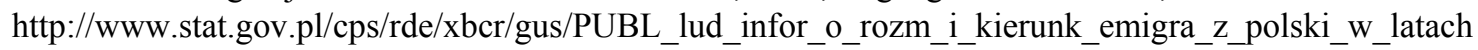
_2004_2010.pdf. 
Abbildung 1 zeigt deutlich, wie die unterschiedliche Politik der zwei alten Mitgliedstaaten in Bezug auf die Übergangsregelungen die Migration von Polen nach Großbritannien positiv und nach Deutschland negativ beeinflusst hat.

Abbildung 1: Polnische Staatsbürger, vorübergehender Aufenthalt in Deutschland und im Vereinigten Königreich (Tsd.)

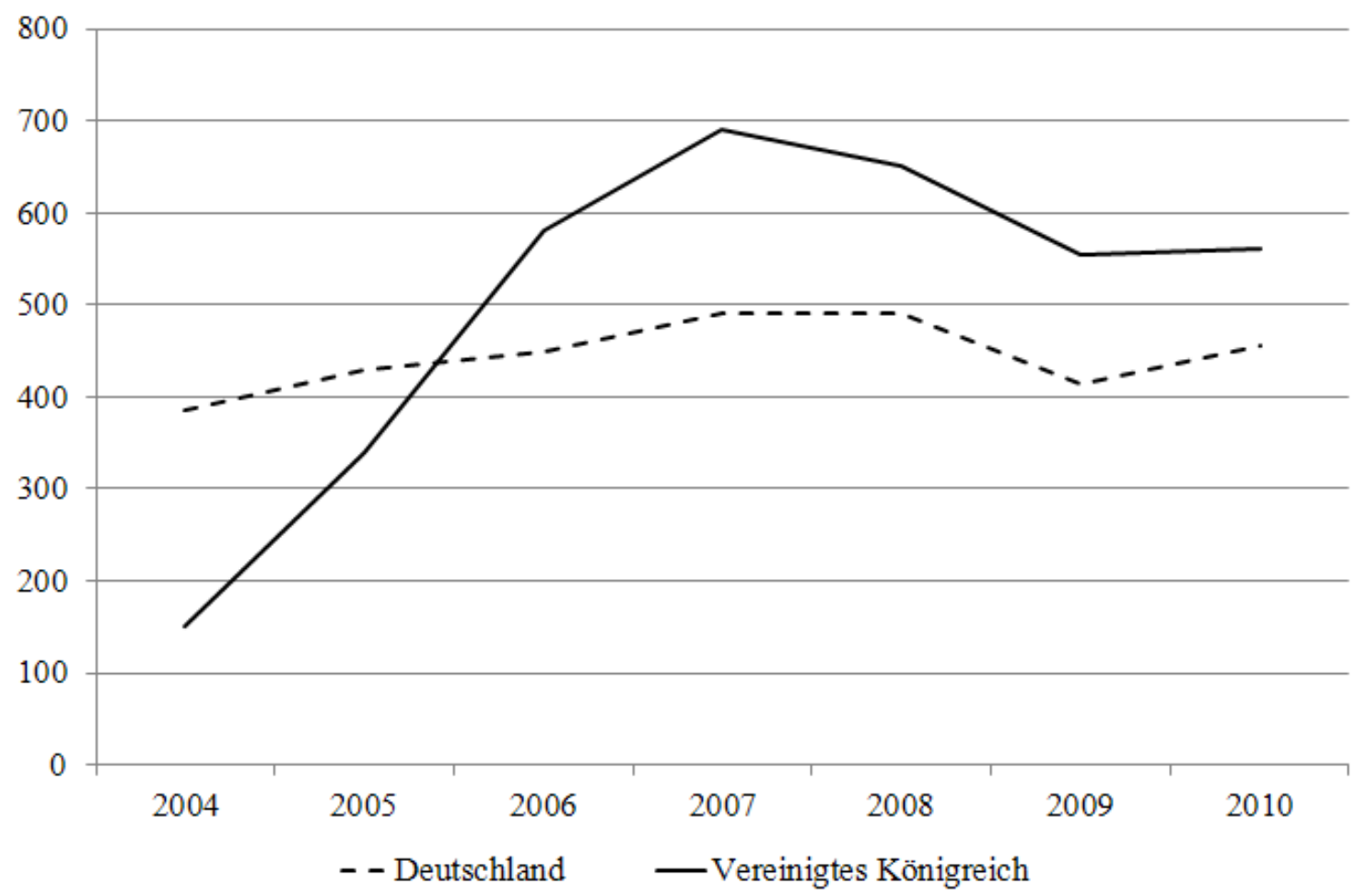

Quelle: Daten aus Tabelle 3.

Innerhalb weniger Jahre erlebte Polen also eine beträchtliche Auswanderung. Der Bevölkerungsnettoabfluss zwischen 2004 und 2009 wird auf 917.000 geschätzt oder 2,4\% der Bevölkerung Polens im Jahr 20047. Für eine Auswanderung entscheiden sich verständlicherweise junge Menschen im arbeitsfähigen Alter, deshalb ist die "Mobilitätsrate" - der Anteil der Bevölkerung im Alter von 15 bis 64 Jahren mit Wohnsitz in anderen EU-Mitgliedstaaten - noch höher: 4,4\% im Jahr $2010^{8}$.

Die jüngste Auswanderung aus Polen nach dem EU-Beitritt unterscheidet sich teilweise von früheren Migrationswellen. Erstens haben die Migranten zum großen Teil neue Ziele gewählt, wie das Vereinigte Königreich oder Irland (Tabelle 3). Zweitens sind die "neuen" Migranten (nach dem EU-Beitritt) jünger als die "alten" (vor dem EU-

7 European Commission, Employment and Social Developments in Europe 2011, Publication Office of the European Union, 2012, S. 252.

8 Ebd., S. 253. Allerdings werden hier auch diejenigen Migranten berücksichtigt, die im Jahr 2010 länger als sieben Jahre den Wohnsitz in einem anderen EU-Mitgliedstaat hatten. 
Beitritt): Zum Beispiel sank das Durchschnittsalter von 30 auf 28 Jahre9. Drittens sind die neuen Auswanderer besser ausgebildet als früher: Zum Beispiel haben etwa 20\% der Migranten einen Hochschulabschluss, im Vergleich zu 14,7\% in der Gesamtbevölkerung Polens im Jahr 200410.

\section{Gewinne und Kosten aus polnischer Sicht}

Wie die Migrationstheorien voraussehen, hat die jüngste Auswanderung aus Polen sowohl Gewinne als auch Kosten verursacht. Die Unterscheidung zwischen den Folgen für die Migranten selbst und für die Gesellschaft bzw. die Volkswirtschaft ist dabei nicht eindeutig, deshalb werden hier sowohl Mikro- als auch Makrowirkungen genannt.

$\mathrm{Zu}$ den Gewinnen der Auswanderung zählen erstens höhere Verdienste bzw. Einkommen. Die Migranten und ihre Familien profitieren somit von einem höheren Lebensstandard. Diese Folge der Migration resultiert direkt aus ihrem wichtigsten Auslöser.

Zweitens fördert die Migration die Mobilität und Aktivität der Menschen. Natürlich zählen diese Merkmale auch zu den Migrationsursachen - die Auswanderungsentscheidung zeigt meistens einen überdurchschnittlichen Unternehmungsgeist. In mehreren Umfragen unter den neuen polnischen Migranten zeigt sich, dass sie nach der Auswanderung noch aktiver und selbstständiger geworden sind.

Drittens nennen die meisten Migranten die Verbesserung der Sprachkenntnisse als den wichtigsten Vorteil der Auswanderung.

Viertens sind auch Geldüberweisungen in die Heimat eine wichtige positive Folge der Migration. Die Überweisungen der polnischen Migranten in Großbritannien nach Polen wurden für 2008 auf 899 Millionen Euro geschätzt, und für 2009 auf 665 Millionen. Insgesamt flossen im Jahr 2010 2,7 Milliarden Euro Nettoüberweisungen nach Polen ${ }^{11}$. Davon profitieren sowohl die Migranten und ihre Familien als auch die Volkswirtschaft. Die Überweisungen gleichen die negative Leistungsbilanz teilweise aus.

Fünftens hat die Auswanderung zum Abbau der Arbeitslosigkeit in Polen beigetragen. Zwar war das hohe Wirtschaftswachstum die wichtigste Ursache für das Sinken der Arbeitslosigkeit, aber die Auswanderung war ein zusätzlicher Faktor. Die Arbeitslosenquote ist von 19,7\% im Jahr 2003 auf 7,1\% im Jahr 2008 gesunken ${ }^{12}$.

9 Fihel u.a., Labour mobility within the EU in the context of enlargement and the functioning of the transitional arrangements, in: Country report Poland, European Integration Consortium, 2009, S. 19.

10 Ebd., S. 19 f.

11 Chukanska/Comini, Economy and finance, Eurostat, Statistics in focus 4/2012, S. 3 f.

12 Eurostat Datenbank, Zugang am 12.03.2012, http://appsso.eurostat.ec.europa.eu/ nui/show.do?dataset=une_rt_a\&lang=de. 
Den Vorteilen stehen allerdings auch Kosten und Probleme der Migration gegenüber. Wieder wird hier sowohl die Mikro- als auch die Makroperspektive eingenommen.

Erstens sind bei jeder Migration mit der Trennung von Familienangehörigen und Freunden psychologische und soziale Kosten bis hin zum Scheitern der Ehe verbunden. Dazu zählt auch eine „doppelte Marginalisierung”, d.h. dass sich die polnischen Migranten sowohl in Polen als auch in ihrem Zuwanderungsland als marginalisiert, oder zumindest nicht voll integriert, fühlen.

Zweitens kommen auch - zumindest in diesem Ausmaß - relativ neue negative soziale Folgen hinzu. So ist der Begriff der „Eurowaisen” eine neue Bezeichnung für das Phänomen, dass in Folge der Auswanderung viele Kinder in Polen ohne einen Elternteil bzw. ohne beide Eltern aufwachsen und zum Beispiel von ihren Großeltern erzogen werden. Nach einem Bericht aus dem Jahr 2008 ist etwa jedes vierte Kind in Polen von dem Phänomen betroffen, dass sich ein Elternteil zu einem zumindest kurzen Arbeitsaufenthalt im Ausland entschlossen hat ${ }^{13}$.

$\mathrm{Zu}$ den größten Problemen der jüngsten Auswanderung aus Polen zählt drittens die Beschäftigung der Migranten unter ihrem Qualifikationsniveau, in der Literatur als brain waste ${ }^{14}$ bezeichnet. Zwar sind die Migranten relativ gut ausgebildet, aber die Arbeit, die sie im Ausland ausüben, entspricht oft nicht ihren Qualifikationen. Es ist durchaus verständlich, dass z.B. auch Akademiker eine einfache Beschäftigung akzeptieren, wenn sie dafür viel besser bezahlt werden als für eine Arbeit in Polen, die ihren Qualifikationen entspräche. Der Schaden hält sich noch in Grenzen, wenn eine solche Beschäftigung nur eine vorübergehende Strategie ist, um z.B. Erfahrungen zu sammeln, Geld zu verdienen oder Sprachkenntnisse zu erweitern. Wenn dieser Zustand aber länger andauert, kommt es zu einer Entwertung von Humankapital und zum Verlust von früher erworbenen Qualifikationen.

Viertens führt das relative hohe Bildungsniveau der Auswanderer zum brain drain in Polen. Natürlich kann man das Ausmaß dieses Problems erst nachträglich beurteilen, weil z.B. eine Rückwanderung das Bild wesentlich verändern würde ${ }^{15}$.

Fünftens hat die Auswanderung das Wirtschaftswachstum in Polen negativ beeinflusst (Abbildung 2).

13 Walczak, Społeczne, edukacyjne i wychowawcze konsekwencje migracji rodziców i opiekunów prawnych uczniów szkół podstawowych, gimnazjalnych i ponadgimnazjalnych (Soziale, pädagogische und erzieherische Folgen der Auswanderung von Eltern und rechtlichen Betreuern von Schülern in Grundschulen und Oberschulen), 2008.

14 Brücker u.a., Labour mobility within the EU in the context of enlargement and the functioning of the transitional arrangements. Final report, European Integration Consortium, 2009, S. 97-103.

15 Ebd., S. 92-97. 
Abbildung 2: Makroökonomische Auswirkungen der Migration aus den neuen Mitgliedstaaten nach dem EU-Beitritt - Herkunfts- und Zielländer (in \%, im Vergleich zu einem hypothetischen Szenario ohne Veränderung von Migrationszahlen und -mustern)

Figure 17. Macroeconomic impacts of post-accession migration from NMS - sending and receiving countries (in \%, as compared to counterfactual scenario assuming no changes in migration scale/patterns)

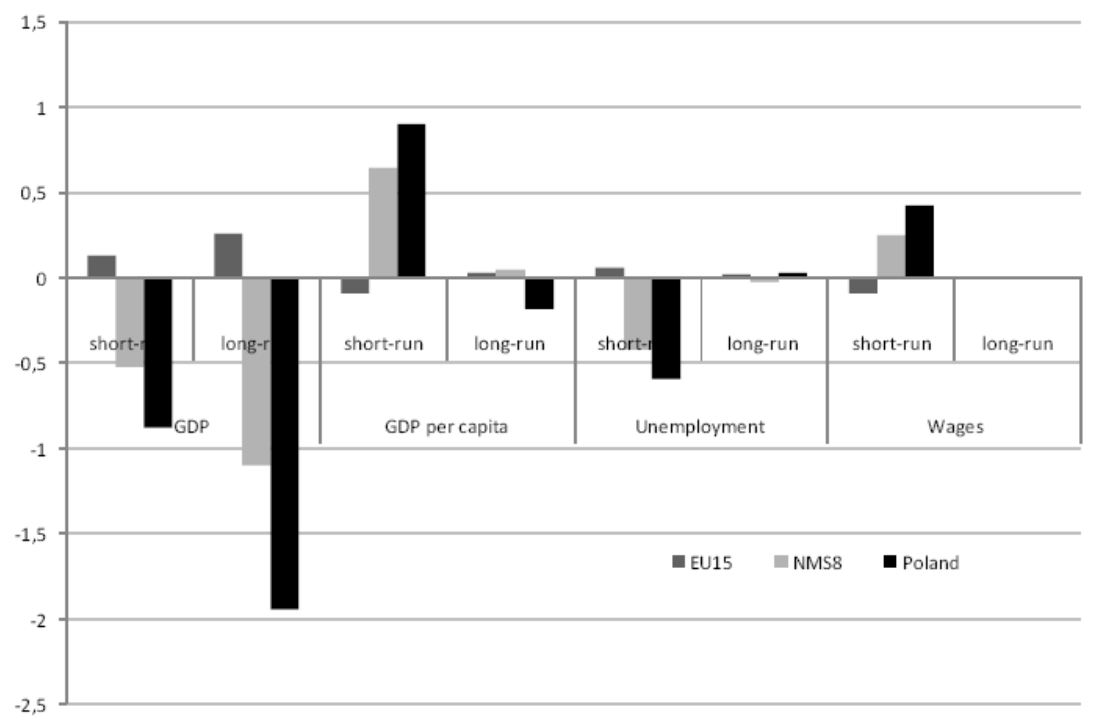

Source: Own elaboration based on Brücker et al. 2009.

Quelle: Fihel (Hrsg.), Recent trends in international migrations in Poland. The 2011 SOPEMI Report, Centre of Migration Research, CMR Working Papers 52/110, University of Warsaw, December 2011, S. 46.

Schließlich hat die Auswanderung von vielen jungen polnischen Bürgern (vgl. Abschnitt 3) die Bevölkerungsalterung in Polen noch beschleunigt. Zum Beispiel entscheiden sich viele polnische Migranten in Großbritannien für Kinder. Ob Polen, dessen Gesamtgeburtenrate $\mathrm{zu}$ den niedrigsten in der EU zählt ${ }^{16}$, davon in Zukunft profitieren wird, hängt wieder von der weiteren Entwicklung der Rückwanderungen ab.

\section{E. Bilanz und Perspektiven}

Wie zuvor erwähnt, ist Migration langfristig sowohl für die Herkunfts- als auch für die Aufnahmeländer günstig. Migration ist natürlich auch ein Ausdruck der Freiheit Menschen sollen immer frei entscheiden dürfen, wo sie leben wollen.

16 2004: 1,23; 2010: 1,38; Quelle: Eurostat Datenbank, Zugang am 08.03.2012, http://epp.eurostat.ec. europa.eu/tgm/table.do?tab=table\&init=1\&language $=$ de $\&$ pcode $=$ tsdde220\&plugin $=1$. 
Eine Migrationsbilanz aus der Perspektive eines Auswanderungslands nach der EUErweiterung im Jahr 2004, d.h. innerhalb eines so kurzen Zeitraums (2004-2011), kann natürlich nur sehr vorsichtig und vorläufig gezogen werden. Mehrmals wurde erwähnt, dass auch die weitere Entwicklung, darunter das Ausmaß der Rückwanderung, für eine "endgültige" Bilanz von zentraler Bedeutung ist.

Für Polen ist die Bilanz insgesamt vorläufig eher enttäuschend. Die negativen Effekte überwiegen die positiven Auswirkungen. Bislang haben viele junge, relativ gut ausgebildete Menschen das Land verlassen, was zu brain drain und brain waste und vielen weiteren negativen Effekten für das Wirtschaftswachstum, die Demographie und die Familien führte.

Die langfristige Perspektive für die Arbeitsmigration sieht für Polen aber anders aus. Dafür gibt es zwei Gründe: Demographie und Konvergenz.

Nach den Bevölkerungsprognosen wird die Bevölkerungszahl Polens von 38,2 Millionen im Jahr 2010 auf 32,7 Millionen im Jahr 2060 sinken. Hat Polen jetzt noch eine junge Bevölkerung, so wird die Alterung schnell voranschreiten und 2060 soll Polen den höchsten Altenquotienten in der EU haben (Tabelle 4). Somit wird Polen also langfristig über kein Auswanderungspotential mehr verfügen und intensiv um Zuwanderung werben müssen.

Tabelle 4: Geschätzter Altenquotient ${ }^{17}$ 2010-2060

\begin{tabular}{|l|c|c|c|c|c|c|}
\hline & 2010 & 2020 & 2030 & 2040 & 2050 & 2060 \\
\hline EU (27 Länder) & 25,92 & 31,37 & 38,33 & 45,52 & 50,16 & 52,55 \\
\hline Deutschland & 31,26 & 35,78 & 47,21 & 56,44 & 58,11 & 59,89 \\
\hline Polen & 18,96 & 26,94 & 35,24 & 39,89 & 53,00 & 64,59 \\
\hline
\end{tabular}

Quelle: Eurostat Datenbank, Zugang am 29.03.2012, http://epp.eurostat.ec.europa.eu/tgm/table. $\mathrm{do} ? \mathrm{tab}=$ table\&init $=1 \&$ language $=$ de $\&$ pcode $=$ tsdde 511 \&plugin $=1$.

Auf der anderen Seite wird der Auswanderungsdruck auch sinken, wenn sich der Prozess der Einkommenskonvergenz zwischen Polen und der EU, der in den letzten 15 Jahren stattgefunden hat (Abbildung 3), weiter fortsetzt.

17 Die Zahl der Personen im Alter von 65 Jahren und älter als Prozentsatz der Zahl der Personen zwischen 15 und 64 Jahren. 


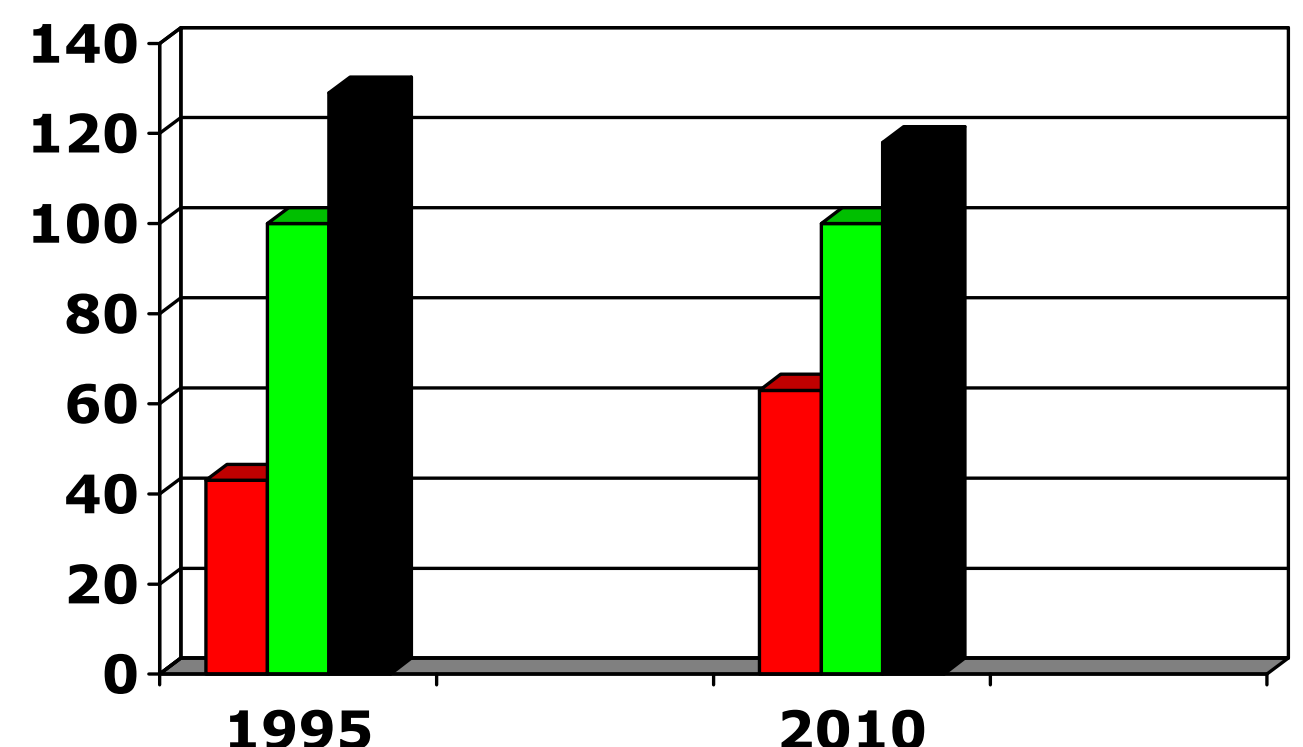

\section{$\square$ PL $\square$ EU $\square$ DE}

Quelle: Eurostat Datenbank, Zugang am 29.03.2012, http://epp.eurostat.ec.europa.eu/ tgm/table.do?tab=table\&plugin $=1 \&$ language $=$ de \&pcode $=$ tec 00114 .

Zusammenfassend lässt sich also feststellen, dass Polen langfristig kein beträchtliches Migrationspotential besitzen wird und es zu einem Abbau der wichtigsten Auswanderungsanreize kommen wird. Dementsprechend soll Polen auch ein Nettozuwanderungsland werden.

Schon vor der Erweiterung im Jahr 2004 haben die Experten festgestellt: „Die bisherigen 15 EU-Länder - allen voran Deutschland und die Länder Südeuropas - werden ihren zukünftigen Bedarf an Zuwanderern im Wesentlichen nicht im Rahmen der zukünftigen Freizügigkeit innerhalb einer erweiterten EU decken können. Auf jene Zuwanderung aus Ostmitteleuropa, vor der sich heute etliche fürchten, werden wir mittelfristig nicht einmal hoffen können“"18.

18 Fassmann/Münz, Auswirkungen der EU-Erweiterung auf die Ost-West-Wanderung, WSI Mitteilungen 2003, S. 25, 32. 


\section{F. Fazit}

Der Beitritt zur Europäischen Union hat zu einem deutlichen Anstieg der Auswanderung aus Polen geführt. Somit wirkte die EU-Integration Polens migrationsfördernd, was in Bezug auf die großen Einkommensunterschiede zwischen Polen und den alten EU-Mitgliedstaaten zu erwarten war.

Dabei unterscheidet sich die Migration nach dem EU-Beitritt sowohl hinsichtlich ihrer Zielrichtungen als auch ihrer Merkmale von der Auswanderung aus Polen vor dem Beitritt. Deutschland wurde durch das Vereinigte Königreich als wichtigstes Zielland ersetzt, was insbesondere auf die unterschiedliche Anwendung der Übergangsfristen für die Arbeitnehmerfreizügigkeit durch die beiden Staaten zurückgeführt werden kann. Die neuen Auswanderer sind auch jünger und besser ausgebildet als die früheren.

Vorübergehend ist die Bilanz der Migration für Polen eher negativ, d.h. die Kosten überwiegen die Gewinne.

Auf längere Sicht wird sich die Situation allerdings ändern. Durch die EUIntegration der neuen Mitgliedstaaten, darunter Polen, sollen die bisherigen Einkommensunterschiede durch Marktintegration und durch zusätzliche EU-Strukturmittel abgebaut werden. Dadurch wird die Abwanderungsbereitschaft sinken.

Die Konvergenz und die schnelle Alterung der Bevölkerung in Polen werden das Auswanderungspotential abbauen und einen wachsenden Bedarf an Zuwanderung erzeugen. 


\title{
Perspektiven eines Europäischen Arbeitsmarktes
}

\author{
Werner Tegtmeier
}

A. Einleitung

B. Ausgewählte, das Arbeitskräftepotential beeinflussende Faktoren 128

$\begin{array}{ll}\text { I. Demographie } & 128\end{array}$

II. Die Komponente der Altersgrenzen $\quad 129$

III. Qualitative Aspekte 131

C. Ausgewählte, die Nachfrage nach Arbeitskräften berührende Aspekte 132

I. Die Lissabon-Strategie - zwischen Anspruch, Wirklichkeit und Notwendigkeiten

II. Arbeitsmarktstabilisatoren 133

III. Arbeitnehmerfreizügigkeit - Wanderungen 134

\section{A. Einleitung}

Nachdenken über mittel- und längerfristige Perspektiven eines Europäischen Arbeitsmarktes heißt, relevante Aspekte zur Entwicklung des Arbeitskräftepotentials und der Nutzung dieses Potentials, der Nachfrage nach Arbeitskräften, zu benennen. Zum 1. Mai 2011 ist die volle Freizügigkeit der Arbeitnehmer auch für die im Jahre 2004 hinzugekommenen Mitglieder der EU wirksam geworden. Und zugleich sind dunkle Wolken für Beschäftigung und Arbeitsmarkt im Gefolge der Staatsschulden- und Finanzkrise aufgezogen, die nicht nur die erzielten beschäftigungspolitischen Fortschritte in Frage stellen, sondern auf Jahre die makroökonomischen Möglichkeiten für mehr Beschäftigung beeinträchtigen werden, und sie werden die bisherige Statik der EU selbst berühren und verändern ${ }^{1}$.

Die von den realwirtschaftlichen und finanziellen Auswirkungen des Zusammenbruchs von Lehman Brothers und ihrer eigenen Staatsverschuldung besonders betroffe-

1 Weder di Mauro, Mitglied des Sachverständigenrates der Bundesregierung zur Begutachtung der gesamtwirtschaftlichen Entwicklung vermutet sogar: „Der Zerfall Europas hat längst begonnen“ (Süddeutsche Zeitung v. 14.11.2011, S. 16) und Bundeskanzlerin Merkel spricht in diesem Kontext von einer „Zeit epochaler Veränderungen in der Welt“ (SZ v. 15.11.11, S. 1), „Wir sind in einer wirklichen systemischen Krise“, Kommissionspräsident Barroso vor dem EU-Parlament in Straßburg (SZ v. 17.11.2011, S. 1). 